\title{
Comparação dos Símbolos OFDM Simulado, Teórico e Medido
}

\author{
Bernardo Valentim e Jair Silva
}

\begin{abstract}
Resumo-Esse artigo apresenta uma comparação entre os sinais OFDM utilizados em modens para comunicação via rede elétrica $(P L C)$. São comparados os sinais gerados através do modelo teórico, da simulação computacional e implementação em FPGA através de VHDL. Os resultados preliminares mostraram boa concordância.
\end{abstract}

Palavras-Chave-OFDM, FPGA, VHDL, PLC.

Abstract-This paper presents a comparison among the theoretical, simulated and implemented OFDM signals for powerline communication $(P L C)$ modems. Preliminary results have shown good agreement.

Keywords-OFDM, FPGA, VHDL, PLC.

\section{INTRODUÇÃO}

Padrão de transmissão das tecnologias DAB (Digital Audio Broadcasting), DVB (Digital Video Broadcasting), WLAN (Wireless Local Area Network), ADSL (Asymmetric Digital Subscriber Line), PLC (Power Line Communications), TV Digital e fortemente cotada a fazer parte do padrão de comunicação móvel da $4^{a}$ geração, a técnica OFDM (Orthogonal Frequency Division Multiplexing) detém valiosas vantagens quando comparada à comunicação com portadora única [1]. A eficiência espectral devido à sobreposição de portadoras, estimação e remoção da dispersão de canal e a reduzida complexidade computacional do transceptor implementado por algoritmos rápidos da IFFT/FFT, são alguns méritos atribuídos à esta técnica. Além disso utiliza o artefato da extensão cíclica para combater a interferência entre símbolos e simplificar a equalização na transmissão em canais com multipercursos [2], [3], [4].

O presente trabalho compara o símbolo OFDM medido na saída de um transmissor, implementado em hardware, com o símbolo OFDM simulado em software e a expressão analítica do mesmo.

O transmissor desenvolvido em hardware, foi concebido em VHDL (VHSIC Hardware Description Language) e implementado em um chip FPGA (Field Programmable Gate Array) Xilinx Virtex-2 xc2v3000 presente no kit Xtreme DSP da Agilent. Este kit conta com conversores A/D (Analógico/Digital) e D/A (Digital/Analógico) de 65 MSPS e 160 MSPS, respectivamente. O projeto foi levado a cabo de modo a permitir maior flexibilidade no que tange à escolha do número de bits de dados, tamanho da IFFT (Inverse Fast Fourier Transform) e do QAM (Quadrature Amplitude Modulation).

Bernardo Valentim e Jair Silva, Laboratório de Telecomunicações, Departamento de Engenharia Elétrica, Universidade Federal do Espírito Santo, Vitória, Brasil, E-mails: bernardolv@gmail.com,djaiscv@ hotmail.com.

\section{Modulação Multiportadora}

A técnica OFDM é uma modulação multiportadora, uma vez que divide o espectro de freqüências em vários subcanais que, modulados em igual número de subportadoras ortogonais, transmite informações simultâneas em subsequiências de informações previamente paralelizadas. Matematicamente, um sinal OFDM complexo em banda base e sem intervalo de guarda na saída do transmissor é dado por,

$$
x(t)=\sum_{i}\left[\sum_{k=0}^{N-1} I_{i, k} e^{j 2 \pi f_{k} t}\right] g\left(t-i T_{B}\right)
$$

onde, os $N$ subsímbolos $\left\{I_{i, k}\right\}_{k=0}^{N-1}$ são transmitidos no bloco $i$, as $N$ senóides complexas $\left\{e^{j 2 \pi f_{k} t}\right\}_{k=0}^{N-1}$ são as subportadoras ortogonais espaçadas em $\frac{1}{T_{B}} H z$, sendo a subportadora $k$ centrada em $f_{k}=\frac{k}{T_{B}}, g(t)$ é o pulso ou janela retangular de amplitude unitária no intervalo de observação $0 \leq t \leq T_{B}$, $T_{B}$ a duração do símbolo OFDM e $j=\sqrt{-1}$. O espectro de frequiências do bloco $i=0$ gerada pela transformada de Fourier da equação 1 é expresso por

$$
X(f)=T_{B} e^{-j 2 \pi f \frac{T_{B}}{2}} \sum_{k=0}^{N-1} I_{0, k} \operatorname{sinc}\left[\left(f-\frac{k}{T_{B}}\right) T_{B}\right]
$$

para $f$ a faixa de freqüências de interesse e a função $\operatorname{sinc}(x)$ igual a 1 para $x=0$ e igual a $\frac{\sin (\pi x)}{\pi x}$ caso contrário [3], [4].

\section{A. Símbolo Simulado}

Para a simulação de um símbolo na saída de um transmissor, considerou-se um sinal de coeficientes reais, ideal para aplicações em sistemas a fio (wireline). Para tanto, foi concebida a simetria Hermitiana nos símbolos complexos na saída da constelação, conforme ilustra o diagrama do transmissor digital da Figura 1.

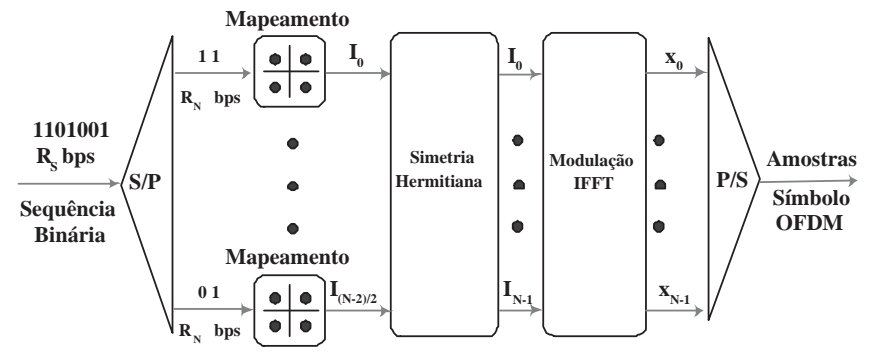

Fig. 1. Diagrama do transmissor OFDM simulado. 
A seqüência de bits que representa os dados a serem transmitidos, são paralelizados e mapeados em $\frac{N-2}{2}$ subsímbolos $I_{k}$ complexos conforme diagrama de constelação pré-definida. A simetria Hermitiana é concebida a estes subsímbolos fazendo $I_{\frac{N}{2}+k}=I_{\frac{N}{2}+k}^{*}$ e $I_{0}=I_{\frac{N}{2}}=0$, feito que faz com que a saída do modulador IFFT seja composta de coeficientes reais. Serializados, tais amostras formam o símbolo OFDM.

\section{B. Símbolo Teórico}

A amostragem no vetor tempo $0 \leq t \leq T_{B}$ do sinal $i=0$ da equação $1 \mathrm{em} N$ intervalos iguais fornece a seqüência

$$
x[i]=\left.x(t)\right|_{t=\frac{i T_{B}}{N}}=\sum_{k=0}^{N-1} I_{k} e^{j 2 \pi \frac{f_{k} i T_{B}}{N}}=\sum_{k=0}^{N-1} I_{k} e^{j 2 \pi \frac{k i}{N}},
$$

que nada mais é que a inversa da transformada discreta de Fourier (IDFT) do vetor de subsímbolos $I_{k}=\left[I_{0}, I_{1}, \ldots, I_{N-1}\right]$. Aplicando-se a simetria Hermitiana à esta seqüencia e efetuando-se alguns algebrismos obtém-se

$$
x(t)=2 \sum_{k=0}^{\frac{N}{2}-1} \Re\left\{I_{k}\right\} \cos \left(\frac{2 \pi k t}{T_{B}}\right)+\Im\left\{I_{k}\right\} \sin \left(\frac{2 \pi k t}{T_{B}}\right),
$$

como expressão analítica para o símbolo OFDM de coeficientes reais.

\section{Símbolo Medido}

Os símbolos, a serem comparados com os sinais gerados pela equação 4 e por uma simulação representada pela Figura 1, foram gerados por um transmissor OFDM. Os símbolos foram capturados do FPGA, que possui conversor D/A em seu kit, por um osciloscópio digital e enviados para um computador conforme se observa na Figura 2.

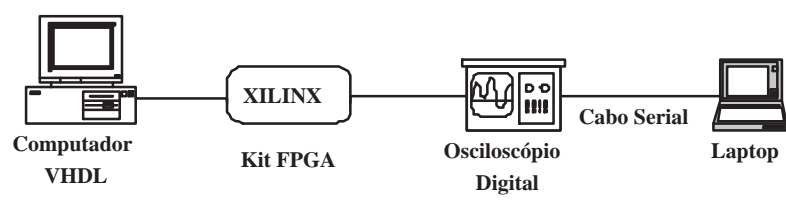

Fig. 2. Esquema de ligação para o símbolo medido.

\section{RESUlTADOS}

Com $\frac{N-2}{2}=31$ subsímbolos $I_{k}$ gerados por uma constelação 4-QAM ( $n=2$ bits por subsímbolo) provenientes de uma seqüencia de bits $=n \times\left(\frac{N-2}{2}\right)=64$ bits, e consequientemente um modulador de $N=64$ pontos ( $N=64$ subsímbolos $x_{i}$ complexos simétricos), a Figura 3 compara os símbolos OFDM simulado, teórico e medido. Os símbolos OFDM, simulado e teórico, foram gerados utilizando a mesma sequência de bits, entretanto, por dificuldade de implementação, o símbolo medido foi gerado aleatóriamente.
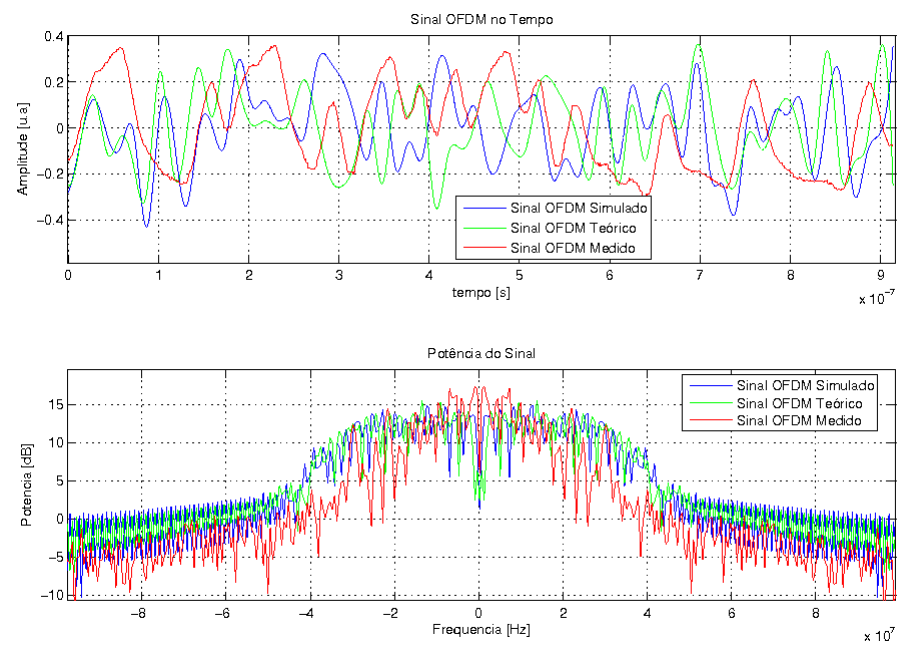

Fig. 3. Comparação dos símbolos OFDM simulado, teórico e medido.

A Figura 3 mostra a excursão dos símbolos de duração útil $T_{u} \cong 914 n s$ e os espectros de amplitude dos mesmos, cujas larguras de banda são $B W \cong 35 \mathrm{MHz}$ para subportadoras espaçadas em $\Delta_{f}=\frac{1}{T_{u}}=\frac{2 B W}{N-1} \cong 1.1 \mathrm{MHz}$. Um sistema multiportadora OFDM de taxa de transferência $R_{s}=\frac{b i t s}{T_{u}} \cong$ $70 \mathrm{Mbps}$ pode ser usado por tais parâmetros. Observa-se ainda pela Figura 3 que embora existe uma diferença na fase dos sinais no tempo, os espectros dos símbolos comparados coincidem.

\section{CONClusões}

Neste trabalho, comparou-se os sinais simulados e teóricos com o obtido por um transmissor OFDM sintetizado em hardware FPGA usando linguagem VHDL. Embora divergentes no domínio do tempo, a semelhança observada nas curvas de espectro de potência, viabilizou o projeto de um transmissor OFDM de $70 \mathrm{Mbps}$, mapeamento 4-QAM e modulador IFFT de 64 pontos, designado a comunicação de dados em canais multipercurso com retardo máximo $\tau=T_{s}-T_{u}=\frac{T_{u}}{0.9}-T_{u} \cong$ $102 n s$.

\section{REFERÊNCIAS}

[1] L. Hanzo, L. Webb, W. Keller Single- and Multi-carrier Quadrature Amplitude Modulation : Principles and Applications for Personal Communications, WLANs and Broadcasting. John Wiley and Sons, Baffins Lane, England, 2000.

[2] J. A. L. Silva, T. M. Siqueira, M. R. N. Ribeiro, R. V. Andreão, E. O. T. Salles, M. E. V. Segatto, A Comparative Analysis of the Performance of OFDM and COFDM Indoor PLC Systems. ICT $2006-13^{\text {th }}$ Conference on Telecommunications, Funchal, v. 1, 2006.

[3] J. A. L. Silva, E. O. T. Salles, M. E. V. Segatto, Análise de Desempenho de um Sistema COFDM para Comunicação de Dados via Rede de Energia Elétrica em Ambientes Internos. $4^{\text {th }}$ International Information and $4^{\text {th }}$ International Information and Telecommunication Technologies Symposium, Florianópolis, 2005.

[4] T. L. Dezan, J. A. L. Silva, K. F. Coco, T. M. Siqueira, E. O. T. Salles, M. E. V. Segatto, Modulação OFDM Aplicada a Power Line Communication. XXI Simpósio Brasileiro de Telecomunicações, Belém/Pa. v. 1. p. 1-6, 2004. 